\title{
REVIEW
}

\section{Elbow examination in the athlete}

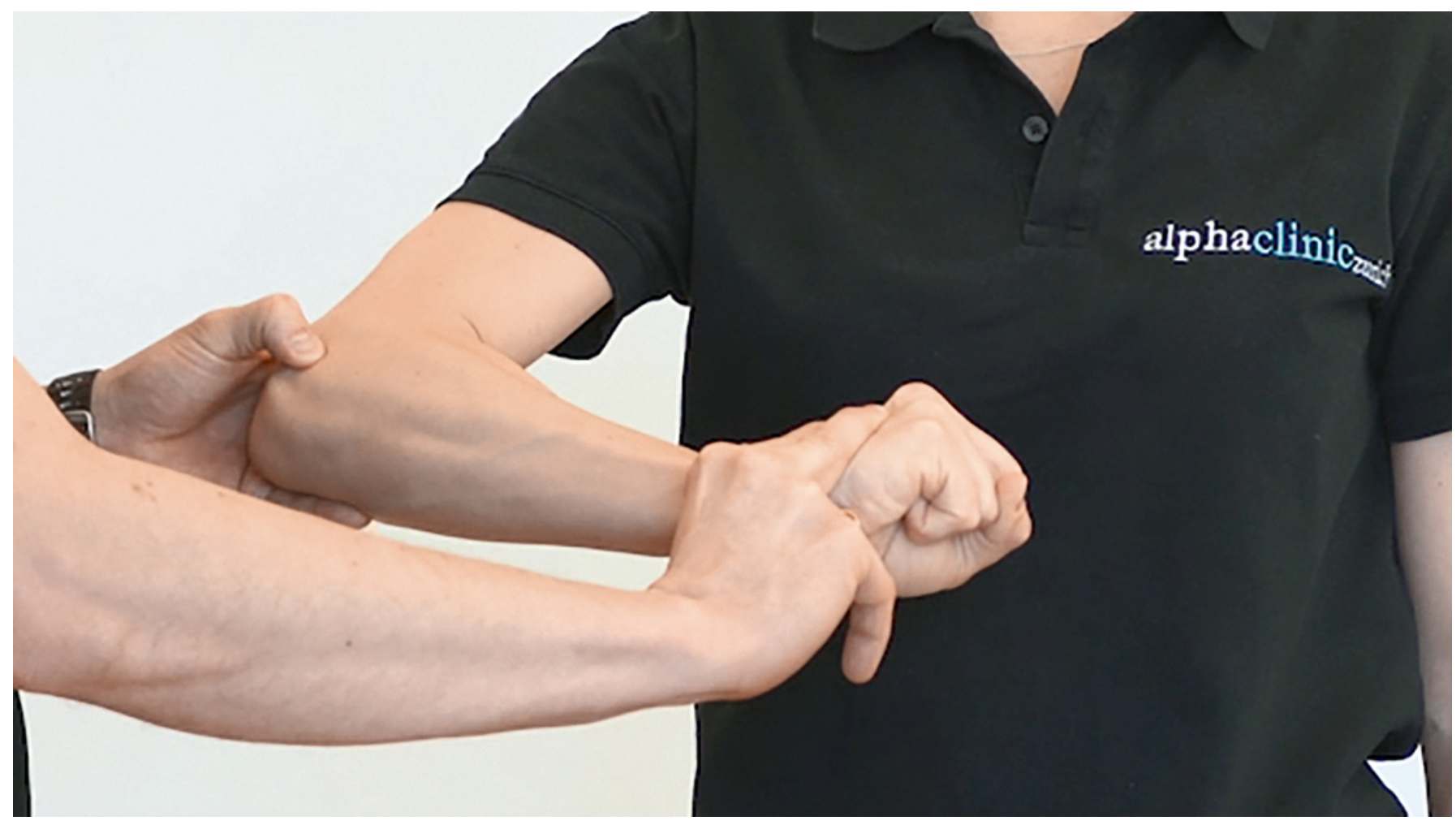

Die Untersuchung des Ellbogens beim Sportler

Vavken $\mathrm{P}^{1},{ }^{2},{ }^{3}$, Rosso $\mathrm{C}^{4},{ }^{5}$

${ }^{1}$ Alphaclinic Zürich, Zürich, CH

${ }^{2}$ ADUS Klinik, Dielsdorf, $\mathrm{CH}$

${ }^{3}$ Division of Sports Medicine, Boston Children's Hospital, Harvard Medical School, Boston, USA

${ }^{4}$ ARTHRO Medics, \#shoulder and elbow center, Basel, $\mathrm{CH}$

${ }^{5}$ Universität Basel, Basel, CH

\section{Abstract}

The elbow is a frequently underestimated and commonly misunderstood joint, leading to a considerable amount of clinical problems. A large part of this due to the largely overlapping and often unspecific symptoms of various diseases and injuries. A better understanding of elbow symptoms and the clinical 
exam of the elbow is the key to unlocking elbow pathology. This paper reviews a comprehensive and concise exam of the elbow that can easily be employed in a primary care sports medicine setting.

\section{Zusammenfassung}

Der Ellbogen ist ein regelmässig unterschätztes und oft missverstandenes Gelenk. Das führt zu einer relevanten Anzahl klinischer Probleme. Die Ursache dafür sind unter anderem die weit überlappenden und oft unspezifischen Symptome verschiedener Erkrankungen und Verletzungen. Ein besseres Verständnis der Symptome des Ellbogens und der klinischen Untersuchung des Ellbogens sind der Schlüssel zur Pathologie des Ellbogens. Die Arbeit beleuchtet einen umfassenden und einfachen Untersuchungsgang für den Ellbogen, der einfach in der sportmedizinischen Primärversorgung eingesetzt werden kann.

\section{Einleitung}

Der Ellbogen ist ein vielfach unterschätztes Problem beim Sportler, auch ausserhalb der Welt des Tennis. Zwei wesentliche Faktoren kommen hier zusammen, einerseits die Tendenz des Ellbogens, auch bei ernsten Verletzungen wenig Probleme zu bereiten, und andererseits auch nur ein sehr enges und überschneidendes Spektrum von Symptomen bei auch sehr verschiedenen Problemen zu zeigen. Es gibt hier aber eine Reihe von red flags in der Anamnese, die die Diagnostik leiten können. [1] (Tabelle 1) Die Mehrzahl der Ellbogenprobleme sind auf chronische Über- oder Fehlbelastung zurückzuführen und können sich bereits im Wachstumsalter äussern. Die klassischen Sportarten sind dabei Radquersport und Sportarten mit repetitiver axialer Belastung, wie Gymnastik oder Gewichtheben. Anatomisch gesehen ist der laterale Ellbogen für Überlastung am vulnerabelsten, im Kindesalter müssen aber auch die multiplen und unregelmässig verteilten Wachstumsfugen berücksichtigt werden.

Traumatische Verletzungen des Ellbogens sind nicht selten. Die rezente Literatur zeigt auch hier, dass oft das Ausmass von Verletzungen unterschätzt wird, aber dass es dafür in der konservativen Therapie eine Tendenz zu Überbehandlung (mit sekundären Schäden) hat [2].

Für diese Arbeit wird der Ellbogen für die Untersuchung in die 4 Quadranten

lateral/ventral/medial/posterior aufgeteilt, um einen einfachen aber strukturierten Untersuchungsablauf zu ermöglichen. Einige Tests werden exemplarisch dargestellt, ein komplettes Video ist auf http://www.derellbogen.ch verfügbar. Wo möglich werden Werte für Sensitivität und Spezifizität angegeben [3].

\section{Anamnese}

Wie bei jeder anderen Untersuchung leitet die Anamnese die nachfolgende Untersuchung des Ellbogens beim Sportler [4]. Die Demographie des Patienten ist ebenso wichtig, wie Art und Dauer der Probleme, Vorerkrankungen oder Unfälle und Vorbehandlungen. Es gibt bei der Erhebung der Anamnese des Ellbogens auch mögliche Pathologien der Schulter und der HWS abzufragen. Bei manchen Instabilitäten kann via Membrana interossea auch das Handgelenk mitbetroffen sein.

Generell sind die Symptome von Ellbogenproblemen eher unverhältnismässig ruhig und fast immer nur (stechender/ziehender) Schmerz, auch bei einem weiten Spektrum an Erkrankungen und Verletzungen. 
Der Ellbogen ist leider kein sehr ausdrucksstarkes Gelenk. Hier gilt es genau zuzuhören und zu hinterfragen. Es hat einige Schlüsselwörter und red flags, die den Untersucher besonders hellhörig machen sollten. (Tabelle 1)

\begin{tabular}{|c|c|c|}
\hline Symptom & Verdacht & Weitere Abklärung \\
\hline $\begin{array}{l}\text { Anhaltender Schmerz >2 Wo nach Radius- } \\
\text { kopffraktur }\end{array}$ & Relevante Begleitverletzung? & CT oder MRT \\
\hline Blockade & Freier Gelenkskörper? (woher?) & Arthro-CT oder MRT \\
\hline $\begin{array}{l}\text { Schmerzbetonung in Flexion/Extension durch } \\
\text { Pronation }\end{array}$ & $\begin{array}{l}\text { Knorpelschaden/Arthrose? } \\
\text { [Pronation schliesst das Gelenk und erhöht } \\
\text { den Anpressdruck des Radius] }\end{array}$ & MRT \\
\hline $\begin{array}{l}\text { Schmerzbetonung in Flexion/Extension durch } \\
\text { Supination }\end{array}$ & $\begin{array}{l}\text { Lateraler Bandschaden? } \\
\text { [Supination entlastet das Gelenk und spannt } \\
\text { die Bänder] }\end{array}$ & US oder MRT \\
\hline $\begin{array}{l}\text { Schmerzbetonung/Krepitus durch Flexion/ } \\
\text { Extension in Varusstress }\end{array}$ & $\begin{array}{l}\text { Fraktur des Coronoid? Posteromediale } \\
\text { Instabilität? }\end{array}$ & $\mathrm{CT}$ \\
\hline Vermeidung von Streckung & Instabilität? Posteromediales Impingement? & CT oder MRT \\
\hline Schmerz im Handgelenk & Verletzung der Membrana interossea/TFCC? & MRT und Rx Handgelenk \\
\hline $\begin{array}{l}\text { Problem beim Armkreisen, Überkopf nach } \\
\text { Sturz auf den gestreckten Arm }\end{array}$ & $\begin{array}{l}\text { Begleitverletzung am posterioren Labrum } \\
\text { der Schulter/hintere Schulterinstabilität? }\end{array}$ & MRT Schulter \\
\hline Anhaltender Schmerz beim Kind/Jugendlichen & Osteochondritis dissecans & $\mathrm{Rx}$ \\
\hline
\end{tabular}

Tabelle 1: Schlüsselwörter in der Anamnese und die Verdachtsdiagnosen

\section{Inspektion}

Auf die Anamnese erfolgt die Inspektion des Ellbogens. Der normale Ellbogen zeigt von vorne einen Valguswinkel zwischen $10^{\circ}$ und $16^{\circ}$. Der Valgus kann auf der dominanten Seite etwas grösser sein. Zeigt sich von vorne ein Varus, so ist dies meist die Folge einer Fraktur während des Kindesalters. Die Inspektion postero-lateral zeigt den Softspot im Dreieck zwischen Epicondylus, Radiuskopf und Olecranon. Ein Erguss des Gelenks kann hier sehr einfach erkannt werden. Die Inspektion von medial ist in der Regel wenig ergiebig, kann aber in Flexion ein Schnappen des N. ulnaris (zw $70^{\circ}$ und $90^{\circ}$ ) und/oder des Triceps (bei $115^{\circ}$ und mehr) zeigen [5]. Anterior zeigt der Ellbogen die Konturen des Biceps und seiner distalen Sehne sowie der Extensoren und Flexoren. Ein Hämatom hier ist ein wichtiger Hinweis auf eine distale Bicepsruptur. In der Betrachtung von posterior zeigt der gebeugte Ellbogen physiologischerweise ein regelmässiges Dreieck, bestehend aus den Epicondylen und dem Olecranon. Die klassische Buristis olecrani ist hier sichtbar.

Die normale Beweglichkeit beträgt in Extension und Flexion $0^{\circ}-140^{\circ}$, aber mit hoher Variabilität. Überstreckbarkeit ist nicht selten, schlanke Individuen können oft mehr beugen, ausgeprägte Muskel können eine Weichteilhemmung verursachen. Die normale Rotation beträgt zwischen $75^{\circ}$ Pronation und $85^{\circ}$ Supination. Für die Funktion des Alltages war bis anhin eine Beweglichkeit von $0^{\circ}-30^{\circ}-130^{\circ}$ als notwendig erachtet worden [6]. Mit der modernen Kommunikation wie der Gebrauch des Natels ist eine 
Flexion von $140^{\circ}$ nötig. Mangelnde Pronation wird durch Abduktion in der Schulter kompensiert. Deswegen ist es wichtig, den Ellbogen bei der Rotationstestung eng am Körper anliegen zu lassen.

\section{Die klinische Testung des Ellbogens}

Der laterale Ellbogen

Der laterale Ellbogen ist die am stärksten belastete und damit verletzlichste Stelle des Gelenks; die meisten Probleme projizieren sich hier. An der Oberfläche gilt es die Extensoren zu testen. Dies geschieht im Cozen Test mit Dorsalextension der geballten, radialduzierten und pronierten Faust und ist positiv bei Schmerzreproduktion am lateralen Epicondylus (Sensitivität 84\%, Spezifizität 0\%). (Abbildung 1) Nota bene ist die Spezifizität in der Literatur 0\% (!), und der Cozen Test ist keine Bestätigung eines Tennisarms, sondern nur einer unspezifischen (0\%!) Überlastung. Die (reflexartige) Verwechslung des lateralen Ellbogenschmerzes mit einem Tennisarm ist eine der Hauptursachen der «therapieresistenten Epicondylitis» [7]. International wird daher inzwischen von «Lateral Elbow Pain» (lateraler Ellenbogenschmerz) gesprochen, den man durch untengenannte Untersuchungen zu differenzieren versucht [8]. Unter den Extensoren liegt der Kapselbandapparat, der mittels Varusstresstest (Endpunkt Schmerzreproduktion: Sensitivität 65\%, Spezifizität 50\%, Endpunkt Aufklappbarkeit: Sensitivität 19\%, Spezifizität 100\%) oder der posterolateralen Schublade gestest wird. Druckdolenz im soft spot weist auf eine hypertrophe Plica dorso-lateralis oder eine Pathologie des Capitulums hin. Der N. radialis kann am lateralen Ellbogen mittels Tinel Zeichen ca. 3-5 cm distal des Epicondylus oder durch Anspannung des Supinator gestest werden. Vorsicht (!), bei der Kompression des R. profundus des N. Radialis kann das EMG/NLG falsch negativ sein [9]. (Tabelle 2)

\section{Der mediale Ellbogen}

Am medialen Ellbogen kann die Flexor-/Pronator-Gruppe per Flexion und Pronation im Provokationstest untersucht werden (Reversed Cozen-Test). Sensitivität und Spezifizität entsprechen dabei dem Cozen-Test. Bis zu 60\% der Flexor- Pronator-Überlastungen haben eine Begleitsymptomatik am N. ulnaris, und dieser wird per Druckschmerz oder Tinel untersucht. In Flexion kann der Nerv schnappen (s.o.), was aber nicht automatisch ein pathologischer Befund ist. Das Innenband wird wie beim Knie durch direkten Valgusstress bei $30^{\circ}$ Flexion getestet (Endpunkt Schmerz: Sensitivität 65\%, Spezifizität 50\%, Endpunkt Aufklappbarkeit: Sensitivität 19\%, Spezifizität 100\%). Zur höheren Sensitivität kann der gebeugte Ellbogen auch auf Schulterhöhe unter Valgusstress gestreckt werden (Moving Valgus Test, Sensitivität 100\%, Spezifizität 75\%). Von weiterer Bedeutung sind medial die Nn. Cutanei antebrachii und brachii medialis. Beide neigen zu sehr schmerzhaften Neuromen, z.B. nach Trauma. Die Diagnose erfolgt durch Anamnese und Tinel, ein hoch-auflösender Ultraschall kann in geübten Händen auch das Neurom zeigen. (Tabelle 3)

\section{Der anteriore Ellbogen}

Der anteriore Ellbogen ist durch den Biceps geprägt. Die Sehne wird im Hook-Test (Abbildung 2) untersucht, wobei eine Fingerspitze unter der Sehne eingehakt wird. Wenn dies nicht geling, ist der Test positiv (Sensitivität 81\%, Spezifizität 100\%). Brachialis und Brachioradialis sind kräftige Beuger am Ellbogen, daher macht Flexion gegen Widerstand wenig Sinn als Testung des Biceps. Viel mehr ist bei Bizepsproblematik (Teilruptur, Bursitis) die Supination schwächer und die Pronation schmerzhaft. Eine 
Medianusreizung kann eine Bizepsproblematik begleiten oder im Rahmen eines sehr seltenen Pronator Teres-Syndroms auftreten. Ist der tiefe Ast betroffen, so können die Patienten kein «OK»-Zeichen geben, da die Flexoren des Daumen und der Finger I und II ausfallen. (Tabelle 4)

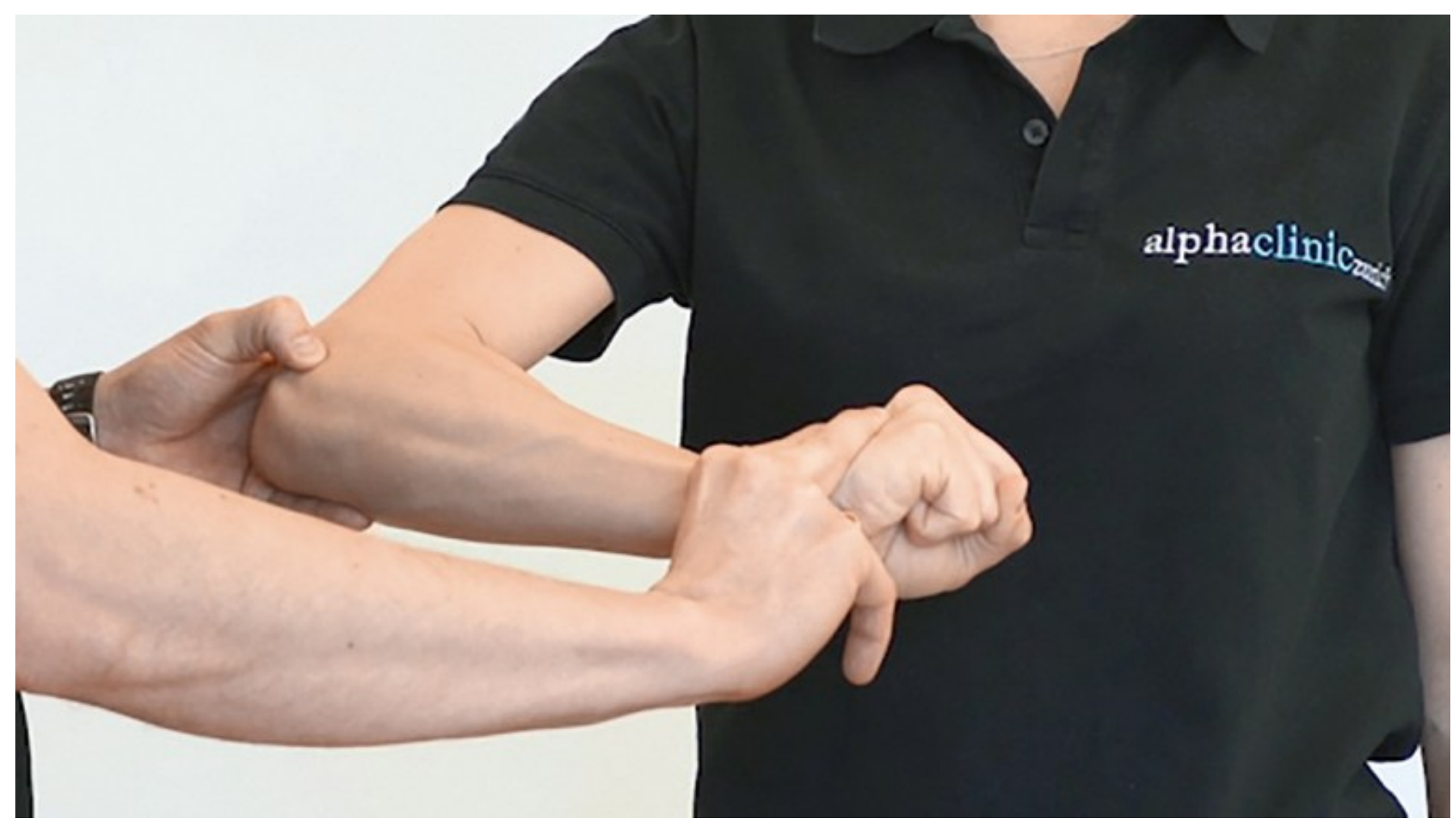

Abbilldung 1 


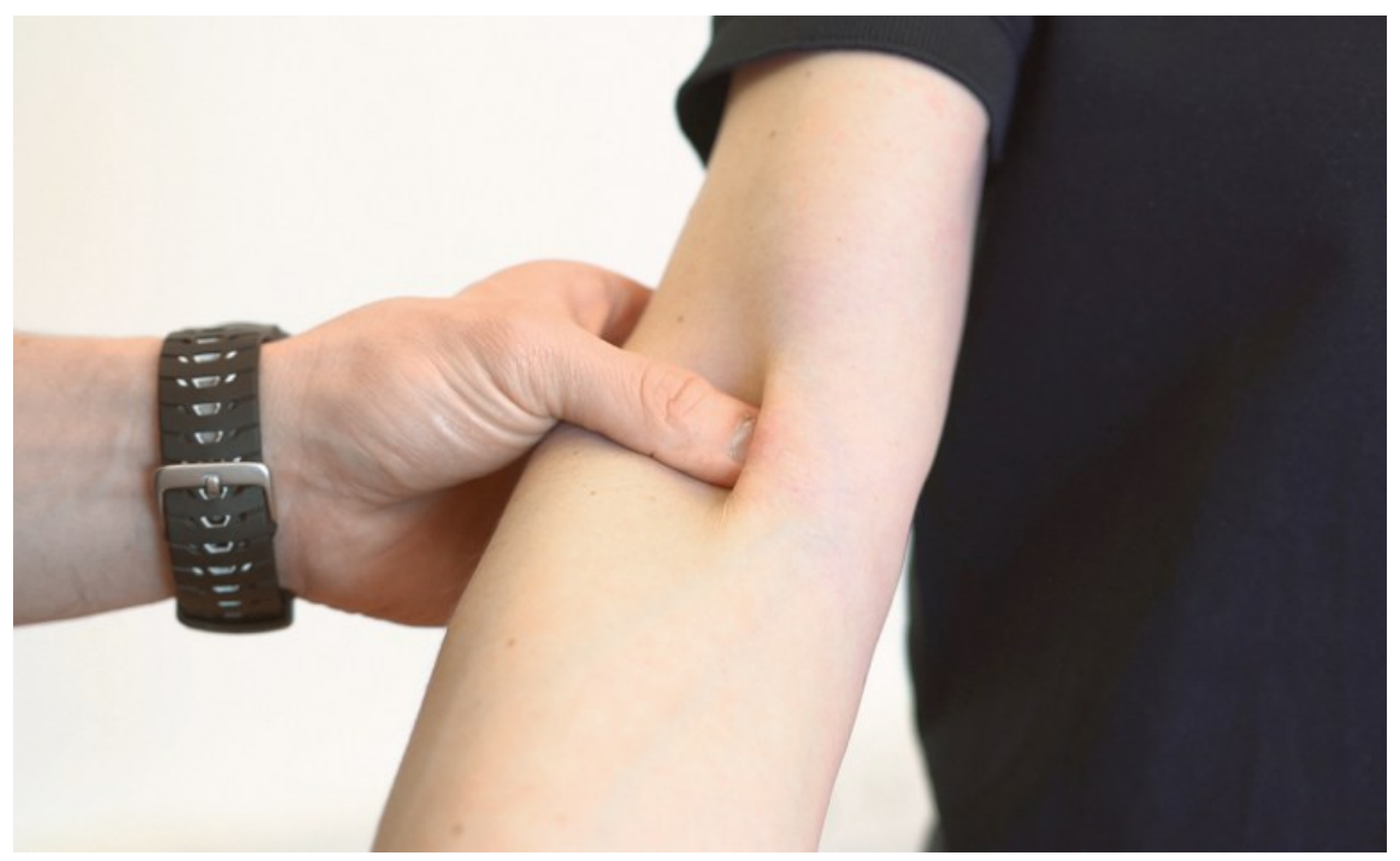

Abbildung 2 


\begin{tabular}{|c|c|c|c|c|c|c|}
\hline DD & Tennisarm & $\begin{array}{l}\text { Extensoren- } \\
\text { ruptur }\end{array}$ & $\begin{array}{l}\text { Seitenband- } \\
\text { instabilität } \\
\text { [PLRI] }\end{array}$ & $\begin{array}{l}\text { Nervenkom- } \\
\text { pression (PIN) }\end{array}$ & $\begin{array}{l}\text { RTRadius- } \\
\text { kopffraktur }\end{array}$ & OCD \\
\hline Ursache & $\begin{array}{l}\text { progressive } \\
\text { Enthesiopathie } \\
\text { der Extensoren. } \\
\text { KEINE Entzün- } \\
\text { dung }\end{array}$ & $\begin{array}{l}\text { \#1 Endstadium } \\
\text { Tennisarm } \\
\text { \#2 Kortison } \\
\text { \#3 Trauma }\end{array}$ & $\begin{array}{l}\text { Defekt lateraler } \\
\text { Bandapparat } \\
\text { [RUHL] } \\
\text { \#1 Kortison } \\
\text { \#2 Trauma }\end{array}$ & $\begin{array}{l}\text { Kompression } \\
\text { des } \mathrm{R} \text {. prof. } \mathrm{N} \text {. } \\
\text { radialis }\end{array}$ & $\begin{array}{l}\text { Auch bei klei- } \\
\text { nem Trauma }\end{array}$ & $\begin{array}{l}\text { Unbekannte } \\
\text { Ursache in v.a. } \\
\text { Knaben 10-16 } \\
\text { Jahre }\end{array}$ \\
\hline $\begin{array}{l}\text { Klinische } \\
\text { Diagnose }\end{array}$ & $\begin{array}{l}\text { Überlastung } \\
\text { Extensoren } \\
\text { [Cozen-Test] \& } \\
\text { Ausschluss } \\
\text { anderer Diag- } \\
\text { nosen }\end{array}$ & Cozen-Test & $\begin{array}{l}\text { Schubladen- } \\
\text { Test } \\
\text { Chair-Test }\end{array}$ & $\begin{array}{l}\text { Kann Zeigefin- } \\
\text { ger nicht } \\
\text { strecken } \\
\text { Schmerzrepro- } \\
\text { duktion durch } \\
\text { Supination gg } \\
\text { Widerstand }\end{array}$ & $\begin{array}{l}\text { DS Radiuskopf } \\
\text { und Schmerz } \\
\text { unter Rotation }\end{array}$ & $\begin{array}{l}\text { \#1 Klinik } \\
\text { \#2 Röntgen }\end{array}$ \\
\hline $\begin{array}{l}\text { Weitere } \\
\text { Diagnostik }\end{array}$ & $\begin{array}{l}\text { Rx [Kalk] } \\
\text { US [Sehnen] } \\
\text { MRT [sek. } \\
\text { Schäden] }\end{array}$ & US oder MRT & US oder MRT & $\begin{array}{l}\text { US mit diagn/ } \\
\text { therapeutischer } \\
\text { Infiltration. } \\
\text { EMG/NLG ev. } \\
\text { falsch negativ }\end{array}$ & $\mathrm{Rx} / \mathrm{CT}$ & MRT \\
\hline $\begin{array}{l}\text { Behandlungs- } \\
\text { optionen }\end{array}$ & $\begin{array}{l}\text { Konservativ: } \\
\text { exzentrische } \\
\text { PT, relative } \\
\text { Ruhe, } \\
\text { Infiltration, } \\
\text { [HA>PRP>Blut] } \\
\text { Operativ: nach } \\
12 \mathrm{Mo}\end{array}$ & $\begin{array}{l}\text { Klinisch symp- } \\
\text { tomatisch } \\
->\text { Refixation }\end{array}$ & $\begin{array}{l}\text { Klinisch symp- } \\
\text { tomatisch - > } \\
\text { Stabilisierung } \\
\text { [konservativ } \\
\text { meist wenig } \\
\text { Erfolg] }\end{array}$ & $\begin{array}{l}\text { \#1 US-Infiltra- } \\
\text { tion reicht in } \\
70 \% \\
\text { \#2 Operative } \\
\text { Dekompression } \\
\text { [bis zu } 18 \mathrm{Mo} \\
\text { Rehab!] }\end{array}$ & $\begin{array}{l}\begin{array}{l}\text { Konservativ } \\
\text { ohne Verschie- } \\
\text { bung }\end{array} \\
\text { ORIF bei Ver- } \\
\text { schiebung/Be- } \\
\text { gleitverletzung }\end{array}$ & $\begin{array}{l}\text { Konservativ: } \\
\text { relative Ruhe, } \\
\text { Belastungs- } \\
\text { verbot, keine } \\
\text { Ruhigstellung } \\
\text { Operativ: ASK }\end{array}$ \\
\hline Red flags & $\begin{array}{l}\text { Sehnenruptur/ } \\
\text { Instabilität } \\
\text { CAVE Cortison }\end{array}$ & Instabilität & $\begin{array}{l}\text { als Tennisarm } \\
\text { getarnt. } \\
\text { Gefahr der frü- } \\
\text { hen Arthrose }\end{array}$ & $\begin{array}{l}\text { +/- motorische } \\
\text { Symptome, in- } \\
\text { direkte Zeichen } \\
\text { am MRT }\end{array}$ & $\begin{array}{l}\text { Anhaltender } \\
\text { Schmerz } \rightarrow \\
\text { Begleitverlet- } \\
\text { zung }\end{array}$ & $\begin{array}{l}\text { Ellbogen- } \\
\text { schmerz beim } \\
\text { Jugendlichen } \\
\text { ernst nehmen! }\end{array}$ \\
\hline
\end{tabular}

Tabelle 2: Differentialdiagnosen LATERALER ELLBOGEN

\section{Der posteriore Ellbogen}

Der Triceps wird durch die Extension gegen Widerstand oder Schwerkraft getestet (Sensitivität und Spezifizität nicht beschrieben), seine Verletzungen sind aber extrem selten. Die Extension kann auch bei einer Tricepsruptur erhalten bleiben, da das Caput medial meist nicht mitrupturiert. Für die Bursitis olecrani braucht es keine besonderen Tests. Nerven hat es am posterioren Ellbogen nicht, der Ulnaris wird zumeist mit dem medialen Ellbogen untersucht. (Tabelle 5)

\section{Der kindliche Ellbogen}

Gerade bei Kindern im Leistungssport ist der Ellbogen hohen Belastungen ausgesetzt und Probleme sind nicht selten [10]. Überlastungen der Wachstumsfugen führen zu schmerzhaften Zuständen wie dem «Little League Elbow», repetitives (Mikro-)Trauma kann Probleme wie eine Osteochondritis dissecans auslösen. 
Letztere kann durch direkten Druck auf das Capitellum untersucht werden, ersterer durch einen Stresstest des Innenbandes und der Flexor-Pronator-Gruppe (Tabelle 6). Die meisten Probleme des kindlichen Ellbogens können erfolgreich konservativ behandelt werden, wenn sie korrekt und zeitgerecht identifiziert werden. Geschieht dies nicht, können ernste und bleibende Schäden entstehen! [11]

\section{Ein kurzes $1 \times 1$ der weiterführenden Diagnostik}

1. Die einfachste weiterführende Untersuchung ist das Röntgen in zwei Ebenen. Das posteriore «Sail Sign» spricht für intraartikuläre Ergüsse oder Hämatome, zumeist wegen Frakturen. Dabei zeigt sich posterior zwischen Humerus und Weichteilen eine Aufhellung.

2. Verkalkungen oder Avulsionen an den Epicondylen sprechen für chronische Sehnen- und Bänderprobleme.

3. Für Kinder kann mit dem Acronym C-R-I-T-O-E (Capitellum-Radius-Innerer Epicondylus-TrochleaOlekranon-Externer/lateraler Epidcondylus) und den ungeraden Zahlen 1-3-5-7-9-11 grob das Erscheinen der Knochenkerne im Nativröntgen leicht im Gedächtnis bleiben.

4. IIn allen Altersstufen sollte die Verlängerung des Radiusschafts in der lateralen Aufnahme auf die Mitte des Capitellum zielen.

5. In Fraktursituationen, speziell bei anhaltenden Schmerzen (>10d), ist das CT sehr hilfreich [12].

6. Wenn es sich um eine chronische Situation handelt, dann kann mit einem Arthro-CT (mit Arthrographie) sowohl Knochen als auch Knorpel hervorragend beurteilt werden. Auch beim Rheumatiker kann mit dem Arthro-CT sowohl Knochen als auch Synovialitis beurteilt werden, vorausgesetzt, das Gelenk wird nicht zu stark mit Kontrastmittel befüllt.

7. Knorpelige freie Gelenkskörper können meist im Arthro- CT besser als im MRT gesehen werden.

8. पDas MRT ist besonders hilfreich, wenn es um Sehnen und Muskeln geht, aber auch zur Beurteilung der Knorpel- und Knochenqualität, z.B. bei einer Osteochondritis dissecans oder einer avaskulären Nekrose. Auch für infektiöse oder neoplastische Prozesse ist das MRT sehr hilfreich. Implantate verursachen aber Artekfakte, die die Diagnose erschweren.

9. ZZunehmend an Bedeutung gewinnt der Ultraschall. Die Untersuchung ist stark vom Können des Untersuchers abhängig, aber erlaubt eine dynamische Beurteilung der Strukturen. Die Auflösung einzelner Sehnen ist besser als im MRT und eine gezielte diagnostisch-therapeutische Infiltration kann durchgeführt werden. Die Eindringtiefe ist aber begrenzt, und Prozesse jenseits der Gelenkskapsel können nur eingeschränkt beurteilt werden.

10. EMG und NLG können in der Diagnostik der Nerven helfen, aber es gibt auch falsch-negative Befunde aufgrund unterschiedlicher Myelinisierung (PIN Kompression!) [7,9]. 


\begin{tabular}{|c|c|c|c|c|c|c|}
\hline DD & Golferellbogen & $\begin{array}{l}\text { Seitenband- } \\
\text { instabilität } \\
\text { [UCL] }\end{array}$ & $\begin{array}{l}\text { Nerven- } \\
\text { kompression } \\
\text { (Ulnaris) }\end{array}$ & $\begin{array}{l}\text { Tardy Ulnar } \\
\text { Nerve Palsy }\end{array}$ & $\begin{array}{l}\text { Posteromedia- } \\
\text { les Impinge- } \\
\text { ment }\end{array}$ & $\begin{array}{l}\text { Posteromedia- } \\
\text { le Rotations- } \\
\text { instabilität }\end{array}$ \\
\hline Ursache & $\begin{array}{l}\text { progressive } \\
\text { Enthesiopathie } \\
\text { Flexor/Pronator } \\
\text { Gruppe. KEINE } \\
\text { Entzündung }\end{array}$ & $\begin{array}{l}\text { Defekt des In- } \\
\text { nenbandes } \\
\text { [UCL] } \\
\text { \#1 Chronisch } \\
\text { \#2 Trauma }\end{array}$ & $\begin{array}{l}\text { Primäre Kom- } \\
\text { pression des } \\
\text { N. Ulnaris }\end{array}$ & $\begin{array}{l}\text { Sekundärer } \\
\text { Schaden des } \\
\text { Ulnaris (durch } \\
\text { Fraktur/UCL/ } \\
\text { Tendinose) }\end{array}$ & $\begin{array}{l}\text { UCL Insuffi- } \\
\text { zienz -> pos- } \\
\text { teromed. } \\
\text { Osteophyten } \\
\text { und Arthrose }\end{array}$ & $\begin{array}{l}\text { Übersehene } \\
\text { Coronoidfraktur } \\
\text { oder terrible } \\
\text { triad }\end{array}$ \\
\hline $\begin{array}{l}\text { Klinische } \\
\text { Diagnose }\end{array}$ & $\begin{array}{l}\text { Flexion/Prona- } \\
\text { tion gg Wider- } \\
\text { stand } \\
\text { Ulnaris! }(>60 \%)\end{array}$ & $\begin{array}{l}\text { Valgus Stress in } \\
30^{\circ} \text { Beugung } \\
\text { Moving Valgus } \\
\text { Apprehension }\end{array}$ & $\begin{array}{l}\text { Anamnese } \\
\text { Tinel } \\
\text { Chronisch: } \\
\text { Pinky escape }\end{array}$ & $\begin{array}{l}\text { Anamnese } \\
\text { Seit-zu-Seit } \\
\text { Vergleich der } \\
\text { Kleinfinger }\end{array}$ & $\begin{array}{l}\text { Posteromedia- } \\
\text { ler DS und me- } \\
\text { diale Aufklapp- } \\
\text { barkeit }\end{array}$ & $\begin{array}{l}\text { Varus Grind } \\
\text { Test }\end{array}$ \\
\hline $\begin{array}{l}\text { Weitere } \\
\text { Diagnostik }\end{array}$ & $\begin{array}{l}\text { Rx (Kalk) } \\
\text { US (Sehne/ } \\
\text { Band) }\end{array}$ & $\begin{array}{l}\text { US } \\
\text { (Arthro) MRT }\end{array}$ & $\begin{array}{l}\text { EMG/NLG } \\
\text { [Nerv] }\end{array}$ & $\begin{array}{l}\text { MRT/CT für } \\
\text { Primär- defekt. } \\
\text { EMG/NLG für } \\
\text { Ulnaris. }\end{array}$ & $\begin{array}{l}\text { MRT (Band) } \\
\text { Ev CT (Phyten) }\end{array}$ & $\begin{array}{l}\text { \#1 Klinik mit } \\
\text { Varus Grind } \\
\text { Test } \\
\text { \#2 CT (Coro- } \\
\text { noid) }\end{array}$ \\
\hline $\begin{array}{l}\text { Behandlungs- } \\
\text { optionen }\end{array}$ & $\begin{array}{l}\text { Konservativ: } \\
\text { exzentrische } \\
\text { PT, relative } \\
\text { Ruhe, } \\
\text { Infiltration } \\
\text { [HA>PRP>Blut] } \\
\text { Operativ: nach } \\
\text { 3-12 Mo }\end{array}$ & $\begin{array}{l}\text { Wird sehr gut } \\
\text { toleriert, nur } \\
\text { die wenigsten } \\
\text { brauchen eine } \\
\text { Operation } \\
\text { [Bandplastik] }\end{array}$ & $\begin{array}{l}\text { \# } 1 \text { Konservati- } \\
\text { ve Therapie } \\
\text { \#2 Operative } \\
\text { Dekompression }\end{array}$ & $\begin{array}{l}\text { \#1 Ursache } \\
\text { behandeln } \\
\text { UND } \\
\text { \#2 Ulnaris- } \\
\text { dekompression }\end{array}$ & $\begin{array}{l}\text { \#1 PT } \\
\text { \#2 Operation } \\
\text { [Dekompres- } \\
\text { sion +/- Stabili- } \\
\text { sierung }\end{array}$ & $\begin{array}{l}\text { Schwierig. } \\
\text { Kann selbst mit } \\
\text { Operation nicht } \\
\text { immer } 100 \% \\
\text { rekonstruiert } \\
\text { werden }\end{array}$ \\
\hline Red flags & $\begin{array}{l}\text { Instabilität } \\
\text { Ulnaris in }>60 \% \\
\text { betroffen }\end{array}$ & $\begin{array}{l}\text { 1) tardy ulnar } \\
\text { nerve palsy } \\
\text { 2) posteromed. } \\
\text { Impingement }\end{array}$ & $\begin{array}{l}\text { DD Kompres- } \\
\text { sion +/- Instabi- } \\
\text { lität } \\
\text { DD Snapping } \\
\text { Triceps }\end{array}$ & $\begin{array}{l}\text { Instabilität, } \\
\text { Fehlstellung } \\
\text { nach Trauma }\end{array}$ & $\begin{array}{l}\text { Mechanische } \\
\text { Beschwerden, } \\
\text { Ulnarisaffektion }\end{array}$ & $\begin{array}{l}\text { Anhaltender } \\
\text { Schmerz nach } \\
\text { Sturz/RK-Frak- } \\
\text { tur! }\end{array}$ \\
\hline
\end{tabular}

Tabelle 3: Differentialdiagnosen MEDIALER ELLBOGEN

\section{Das Wichtigste für die Praxis}

Der Ellbogen ist ein ausdrucksschwaches Gelenk und hat sehr ähnliche und unverhältnismässig geringe Symptome für eine Fülle von Problemen.

Der laterale Ellbogenschmerz ist ein unspezifisches Symptom, das in vielen Krankheitsbildern ausser dem Tennisarm vorkommt.

Ein chronischer, therapieresistenter Tennisarm (>6 Mo) ist in 3 von 4 Fällen kein Tennisarm, sondern ein anderes Problem.

Gerade bei Kindern sind Überlastungsschäden am Ellbogen ein wachsendes Problem, können bei zeitgerechter Diagnose aber meist gut konservativ behandelt werden. 


\begin{tabular}{|c|c|c|c|c|c|}
\hline DD & Bicepstendinitis & Bicepsruptur & $\begin{array}{l}\text { Anteriore Kapsel- } \\
\text { zerrung }\end{array}$ & Pronatorsyndrom & Arthrose \\
\hline Ursache & $\begin{array}{l}\text { Überlastung } \\
\text { Biceps, auch } \\
\text { Partialruptur }\end{array}$ & $\begin{array}{l}\text { Trauma loft wenig } \\
\text { spektakulär) mit } \\
\text { sofortigem } \\
\text { Schmerz und } \\
\text { Hämatom }\end{array}$ & $\begin{array}{l}\text { Repetitive oder } \\
\text { forcierte Hyperex- } \\
\text { tension }\end{array}$ & $\begin{array}{l}\text { Kompression des } \\
\text { Nervus Medianus }\end{array}$ & $\begin{array}{l}\text { \#1 Posttrauma- } \\
\text { tisch lauch kleine } \\
\text { Unfälle!!!! } \\
\text { \#2 Rheuma } \\
\text { \#3 Degenerativ }\end{array}$ \\
\hline $\begin{array}{l}\text { Klinische } \\
\text { Diagnose }\end{array}$ & $\begin{array}{l}\text { DS Tuberositas } \\
\text { Radii, Belastungs- } \\
\text { schmerz Biceps }\end{array}$ & $\begin{array}{l}\text { Hook Test } \\
\text { Hämatom antero- } \\
\text { medial wenn frisch }\end{array}$ & $\begin{array}{l}\text { Anteriorer } \\
\text { Schmerz nach } \\
\text { Hyperextension \& } \\
\text { Ausschluss ande- } \\
\text { rer Ursachen }\end{array}$ & $\begin{array}{l}\text { Schmerzrepro- } \\
\text { duktion durch for- } \\
\text { cierte Pronation }\end{array}$ & $\begin{array}{l}\text { Anamnese, Krepi- } \\
\text { tus unter Flexion/ } \\
\text { Extension mit } \\
\text { Faustschluss }\end{array}$ \\
\hline $\begin{array}{l}\text { Weitere } \\
\text { Diagnostik }\end{array}$ & $\begin{array}{l}\text { \#1 Klinik } \\
\text { \#2 MRT oder US }\end{array}$ & $\begin{array}{l}\text { Klinik reicht eig. } \\
\text { MRT/US foren- } \\
\text { sisch }\end{array}$ & $\begin{array}{l}\text { Rx wg mgl Myositis } \\
\text { ossificans (Brachi- } \\
\text { alis) } \\
\text { MRT bei anhalten- } \\
\text { den Beschwerden }\end{array}$ & $\begin{array}{l}\text { \#1 MRT } \\
\text { \#2 MG/NLG [oft } \\
\text { inkonklusiv] }\end{array}$ & $\begin{array}{l}\mathrm{RX} \\
\mathrm{CT}\end{array}$ \\
\hline $\begin{array}{l}\text { Behandlungs- } \\
\text { optionen }\end{array}$ & $\begin{array}{l}\text { Konservativ mit } \\
\text { PT, relative Ruhe, } \\
\text { Infiltration. } \\
\text { Sehr selten eine } \\
\text { Op nötig (bei } \\
\text { Partialruptur) }\end{array}$ & $\begin{array}{l}\text { \#1 Konservativ für } \\
\text { "low-demand" } \\
\text { (-40\% Supination/ } \\
-30 \% \text { Flexion/ } \\
-20 \% \text { Faust- } \\
\text { schluss) } \\
\text { \#2 Refixation }\end{array}$ & PT & $\begin{array}{l}\text { \#1 PT für } 6 \text { Monate } \\
\text { \#2 Medianus- } \\
\text { dekompression } \\
{[-80 \% \text { Erfolg] }}\end{array}$ & $\begin{array}{l}\text { \#1 konservativ } \\
\text { \#2 operativ mit } \\
\text { Gelenkserhalt } \\
\text { \#3 Prothese }\end{array}$ \\
\hline Red flags & $\begin{array}{l}\text { Schmerz in der } \\
\text { Tiefe neben der } \\
\text { Sehne } \rightarrow \text { Coronoid }\end{array}$ & $\begin{array}{l}\text { \#1 "Pop" } \\
\text { \#2 Hämotom } \\
\text { medial }\end{array}$ & $\begin{array}{l}\text { Gleichzeitige } \\
\text { Bandverletzung } \\
\text { oder Fraktur }\end{array}$ & $\begin{array}{l}\text { DD Karpaltunnel } \\
\text { Cave: double crush }\end{array}$ & $\begin{array}{l}\text { Prothetik letzte } \\
\text { Option, gelenks- } \\
\text { erhaltende Ops } \\
\text { nicht übersprin- } \\
\text { gen! }\end{array}$ \\
\hline
\end{tabular}




\begin{tabular}{|c|c|c|c|}
\hline DD & Tricepstendinose & $\begin{array}{l}\text { Posteromediales Impinge- } \\
\text { ment }\end{array}$ & Bursitis Olecrani \\
\hline Ursache & $\begin{array}{l}\text { Chronische Überlastung. } \\
\text { Postoperativ (ASK-Portale) }\end{array}$ & $\begin{array}{l}\text { UCL Insuffizienz } \rightarrow \text { postero- } \\
\text { med. Osteophyten und Ar- } \\
\text { throse }\end{array}$ & $\begin{array}{l}\text { Reizung } \\
\text { Infekt }\end{array}$ \\
\hline Klinische Diagnose & $\begin{array}{l}\text { DS und Schmerz bei } \\
\text { Streckung gg Widerstand }\end{array}$ & $\begin{array}{l}\text { Posteromedialer DS und } \\
\text { mediale Aufklappbarkeit }\end{array}$ & Blickdiagnose \\
\hline Weitere Diagnostik & $\begin{array}{l}\text { Rx [Alignement] } \\
\text { US oder MRT [Struktur- } \\
\text { schäden] }\end{array}$ & $\begin{array}{l}\text { MRT (Band) } \\
\text { Ev. CT [Phyten] }\end{array}$ & $\begin{array}{l}\text { Infekt: Blut/Punktat } \\
\text { Chronische Reizung: Rx } \\
\text { Knochenunregelmässigkeit }\end{array}$ \\
\hline Behandlungsoptionen & $\begin{array}{l}\text { \#1 PT } \\
\text { \#2 Infiltration } \\
\text { \#3 Operativ bei (Partial-) } \\
\text { Ruptur }\end{array}$ & $\begin{array}{l}\text { \#1 PT } \\
\text { \#2 Operation (Dekompres- } \\
\text { sion +/- Stabilisierung }\end{array}$ & $\begin{array}{l}\text { \#1 Ruhigstellung } \\
\text { \#2 Aspiration/Infiltration } \\
\text { \#3 Bursektomie }\end{array}$ \\
\hline Red flags & Streckschwäche & $\begin{array}{l}\text { Mechanische Beschwerden, } \\
\text { Ulnarisaffektion }\end{array}$ & $\begin{array}{l}\text { Studien zeigten keinen Un- } \\
\text { terschied zW Aspiration +/- } \\
\text { Infiltration und Ruhigstellung } \\
\text { allein }\end{array}$ \\
\hline
\end{tabular}




\begin{tabular}{|c|c|c|c|c|c|c|}
\hline DD & $\begin{array}{l}\text { Osteochondritis } \\
\text { Dissecans (OCD) }\end{array}$ & Morbus Panner & $\begin{array}{l}\text { Little League } \\
\text { elbow }\end{array}$ & $\begin{array}{l}\text { Bandverlet- } \\
\text { zung (lateral/ } \\
\text { medial) }\end{array}$ & Chassaignac & Frakturen \\
\hline Ursache & $\begin{array}{l}\text { Unklar [Überlas- } \\
\text { tung/Perfusion) }\end{array}$ & $\begin{array}{l}\text { Unklar [OCD } \\
\text { der Jungen?] }\end{array}$ & $\begin{array}{l}\text { Mediale Über- } \\
\text { lastung mit } \\
\text { Apophysitis bis } \\
\text { Avulsion des } \\
\text { Epicondylus }\end{array}$ & $\begin{array}{l}\text { Auch beim Kind } \\
\text { möglich! }\end{array}$ & $\begin{array}{l}\text { Radiuskopfluxa- } \\
\text { tion durch axia- } \\
\text { len Zug [«Kin- } \\
\text { dermädchen»] }\end{array}$ & $\begin{array}{l}10 \% \text { der kindli- } \\
\text { chen Frakturen } \\
\text { am Ellbogen }\end{array}$ \\
\hline $\begin{array}{l}\text { Klinische } \\
\text { Diagnose }\end{array}$ & $\begin{array}{l}\text { Lateraler Ell- } \\
\text { bogenschmerz } \\
\text { beim } 11-15-\mathrm{j} \text {. } \\
\text { Patient. Evtl. } \\
\text { mechanische } \\
\text { Symptome } \\
\text { DS am Capitel- } \\
\text { lum }\end{array}$ & $\begin{array}{l}\text { Wie OCD, aber } \\
<10 \text { Jahre alt }\end{array}$ & $\begin{array}{l}\text { DS medial. } \\
\text { Schmerzrepro- } \\
\text { duktion durch } \\
\text { Flexion gg Wi- } \\
\text { derstand und } \\
\text { Valgusstress. } \\
\text { Evtl. Flexions- } \\
\text { defizit }\end{array}$ & $\begin{array}{l}\text { Aufklappbarkeit } \\
\text { in den Stress- } \\
\text { Tests }\end{array}$ & $\begin{array}{l}\text { Anamnese und } \\
\text { Pseudoparese }\end{array}$ & $\begin{array}{l}\text { Sturzanamnese } \\
\text { Schmerz } \\
\text { Schwellung }\end{array}$ \\
\hline $\begin{array}{l}\text { Weitere } \\
\text { Diagnostik }\end{array}$ & $\begin{array}{l}\text { Rx [Diagnose] } \\
\text { MRT [Verlauf] }\end{array}$ & $\begin{array}{l}\text { Rx (Diagnose) } \\
\text { MRT (Verlauf) }\end{array}$ & $\begin{array}{l}\text { Rx (Fuge) } \\
\text { MRT (KM } \\
\text { Ödem] }\end{array}$ & US oder MRT & $\begin{array}{l}\text { Wenn Repo } \\
\text { nicht gelingt, } \\
\text { Rx wg Fraktur }\end{array}$ & $\begin{array}{l}\text { Röntgen } \\
\text { [posteriores } \\
\text { Sail Sign!] }\end{array}$ \\
\hline $\begin{array}{l}\text { Behandlungs- } \\
\text { optionen }\end{array}$ & $\begin{array}{l}\text { Konservativ: } \\
\text { «Bewegung darf } \\
\text { sein, Belastung } \\
\text { nein» [50\%-80\% } \\
\text { Erfolg] } \\
\text { Operativ }\end{array}$ & $\begin{array}{l}\text { Primär konser- } \\
\text { vativ, Operation } \\
\text { primär kon- } \\
\text { traindiziert }\end{array}$ & Konservativ & $\begin{array}{l}\text { Konservativ [?] } \\
\text { Wachstums- } \\
\text { fugenschonen- } \\
\text { de Bandplastik }\end{array}$ & $\begin{array}{l}\text { Reposition z.B. } \\
\text { durch Supina- } \\
\text { tion/Flexion } \\
\text { oder Hyper- } \\
\text { pronation } \\
\text { [etwas besser] }\end{array}$ & $\begin{array}{l}\text { Frakturabhän- } \\
\text { gig, aber sehr } \\
\text { oft konservativ }\end{array}$ \\
\hline Red flags & $\begin{array}{l}\# 1 \text { Mechanische } \\
\text { Symptome } \\
\# 2 \text { Streckdefizit } \\
>20^{\circ} \\
\# 3 \text { geschlossene } \\
\text { Fugen am Rx }\end{array}$ & Wie OCD & $\begin{array}{l}\text { Innenrotations- } \\
\text { defizit der } \\
\text { Schulter } \\
\text { ipsilateral }\end{array}$ & $\begin{array}{l}\text { Kann beim Kind } \\
\text { schnell in eine } \\
\text { Arhtrofibrose } \\
\text { umschlagen }\end{array}$ & $\begin{array}{l}\text { Lig. Annulare } \\
\text { als Repositions- } \\
\text { hindernis } \\
\text { In chron. Fällen } \\
\text { Redressions- } \\
\text { gips }\end{array}$ & $\begin{array}{l}\text { Luxationen } \\
\text { Olekranonfrak- } \\
\text { tur bei Osteo- } \\
\text { gensis imper- } \\
\text { fecta }\end{array}$ \\
\hline
\end{tabular}

\section{Acknowledgments, conflict of interest and funding}

No conflict of interest to be declared. No funding was obtained.

\section{Corresponding author $\square$}


Patrick Vavken

PD Dr. med.

alphaclinic Zürich

Kraftstrasse 29, 8044 Zürich

0443888411

vavken@alphaclinic.ch

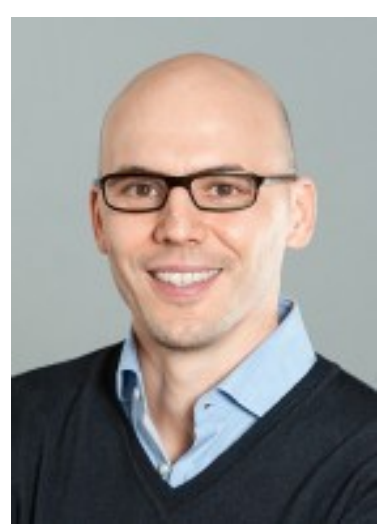

\section{References}

1. Vavken P, Rosso C. Der schmerzende Ellbogen in der Praxis. Schweiz Med Forum. 2017;17(44):953-9.

2. Vavken P, Vavken J, Demarmels S, Rikli D. [Associated Injuries in Radial Head Fractures]. Z Orthopadie Unfallchirurgie. 2017 Apr; 155(2):220-5.

3. Zwerus EL, Somford MP, Maissan F, Heisen J, Eygendaal D, van den Bekerom MP. Physical examination of the elbow, what is the evidence? A systematic literature review. Br J Sports Med. 2018 Oct;52(19):1253-60.

4. Hsu SH, Moen TC, Levine WN, Ahmad CS. Physical examination of the athlete's elbow. Am J Sports Med. 2012 Mar;40(3):699-708.

5. Rioux-Forker D, Bridgeman J, Brogan DM. Snapping Triceps Syndrome. J Hand Surg. 2018;43(1):90.e1-90.e5.

6. Morrey BF, Askew LJ, Chao EY. A biomechanical study of normal functional elbow motion. J Bone Joint Surg Am. 1981 Jul;63(6):872-7.

7. Vavken P. Wenn ein Tennisarm kein Tennisarm ist ... Differenzialdiagnosen des lateralen Ellbogenschmerzes. Prax Bern. 106(1):29-36.

8. Vavken P. Evidenzbasierte Behandlung des lateralen Ellbogenschmerzes. Lead Opin. 2018;3:62-5.

9. Moradi A, Ebrahimzadeh MH, Jupiter JB. Radial Tunnel Syndrome, Diagnostic and Treatment Dilemma. Arch Bone Jt Surg. 2015 Jul; 3(3):156-62.

10. Vavken P, Müller AM, Camathias C. First 50 Pediatric and Adolescent Elbow Arthroscopies: Analysis of Indications and Complications. J Pediatr Orthop. 2016 Jun;36(4):400-4.

11. Vavken P. Ellbogenbeschwerden des adoleszenten Sportlers. Lead Opin. 2019;1:6-9.

12. Vavken P. Doch keine einfache Radiuskopffraktur ... Prax Bern. 2017; 106(6):319-21. 